\title{
ENDOVASCULAR INFRARENAL AORTIC ANEURYSM REPAIR COMBINED WITH LAPAROSCOPIC CHOLECYSTECTOMY
}

\author{
doi: $10.1590 / \mathbf{S 1 8 0 7 - 5 9 3 2 2 0 1 0 0 0 0 7 0 0 0 1 5}$
}

Nelson Wolosker, Cynthia de Almeida Mendes, Carlos Eduardo Jacob, Angela Maria Borri Wolosker, Pedro Puech-Leão

\section{INTRODUCTION}

The incidence of concomitant abdominal aortic aneurysm (AAA) and asymptomatic cholelithiasis is $5 \%$ to $6 \% .{ }^{1} \mathrm{In}$ such situations, isolated standard AAA repair is associated with acute cholecystitis in up to $18 \%$ of these concomitant cases, ${ }^{2}$ which justifies correcting these two diseases together. When a median route is used, it can lead to infection of the prosthesis. A good alternative for preventing this complication is the use of endovascular techniques that are rarely associated with perioperative cholecystitis ${ }^{3}$ or the use of two different surgical routes during the same operation. ${ }^{4}$

With the development of endovascular and videolaparoscopic techniques, a further possibility described in this case report enables correction of the two diseases in the same operation with the aim of diminishing the risk of infection: endovascular treatment of infrarenal AAA associated with laparoscopically assisted cholecystectomy.

\section{CASE REPORT}

A 66-year-old man was found to have an asymptomatic 5.7-cm infrarenal AAA and cholelithiasis on a computed tomography (CT) scan of his abdomen. He had a history of controlled systemic arterial hypertension, myocardial infarction (14 years previously), coronary revascularization (one year previously) and chronic kidney failure. He was undergoing hemodialysis three days a week and awaiting a kidney transplant.

An infrarenal AAA measuring $5.7 \mathrm{~cm}$ was found while investigating a condition of abdominal pain in the

Disciplina de Cirurgia Vascular, Hospital das Clínicas, Faculdade de Medicina, Universidade de São Paulo - São Paulo/SP, Brazil.

Email: cynthiamendes35@hotmail.com

Tel.: 5511 3885-5361 right hypochondrium. On this occasion, chronic calculous cholecystopathy was also diagnosed.

Initially, the patient underwent endovascular treatment of AAA, using a Talent bifurcated endoprosthesis measuring 24 x 12 x $140 \mathrm{~mm}$ (main body), 14 x 12 x $75 \mathrm{~mm}$ (main body extension) and $12 \times 12 \times 90 \mathrm{~mm}$ (left branch) by dissection of the bilateral femoral arteries under general anesthesia. The operation lasted 85 minutes, $180 \mathrm{ml}$ of iodide contrast agent was used, and there were no complications. After closure of the skin and reversion of anticoagulation, the patient underwent laparoscopy-assisted cholecystectomy. During the procedure, gallbladder empyema was observed, and, when drained, it caused extravasation of pus into the abdominal cavity. The bile was sent for bacteriological analysis. Cholecystectomy and intra-operative cholangiography were successfully performed, and a drain was left in the subhepatic space. Empirical antibiotic therapy consisting of endovenous ceftriaxone and metronidazole was started during the operation.

The patient demonstrated good postoperative evolution and did not require vasoactive drugs. He underwent hemodialysis soon after the surgical procedure and had the tubes removed in the operation room. Nasogastric intubation was stopped and fluid intake was started after 24 hours. Food intake was started 12 hours later. The drain in the right hypochondrium was removed on the fourth day after the operation.

Multisensitive Escherichia coli was identified in the liquid aspirated from the gallbladder. Oral ciprofloxacin treatment was therefore started, and this was maintained for 14 days.

The patient's postoperative course was free of complications, and he was discharged from the hospital on the seventh day after the operation. One month after the surgery, the patient had resumed a normal life.

A control CT scan taken six months after the operation is presented in Figure 1. 


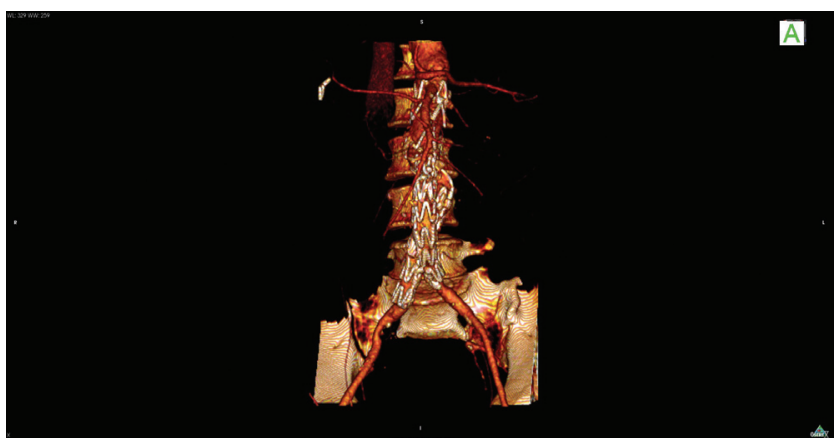

Figue 1 - Control CT Scan

\section{DISCUSSION}

The surgical approach used with patients having AAA and cholelithiasis is controversial. If we choose to treat only the aneurysm by an intraperitoneal route, the patient may present acute cholecystitis during the immediate postoperative period, ${ }^{1,5}$ although endovascular techniques reduce this complication. ${ }^{3}$ If we treat the cholecystopathy alone, the aneurysm might rupt after the operation because of collagenase activity on the aortic wall following the intra-abdominal procedure. ${ }^{7}$ Simultaneous repair through an intraperitoneal route may cause infection in the prosthesis, ${ }^{5}$ especially in cases such as the one discussed in this paper, in which silent acute cholecystitis (oligosymptomatic) was diagnosed only during the operation, along with extravasation of its infected content. If the procedure had been carried out using an intraperitoneal route, the prosthesis would certainly have become contaminated. Due to the technique used in this case, the gallbladder contents did not come into contact with the prosthesis at any time, because the two accesses used were not directly accessible to each other. Thus, there was no contamination of the prosthesis and, consequently, no infectious complications. The endovascular repair of aneurysms appears to have a minor incidence of acute cholecystitis in the perioperative period, ${ }^{3}$ but in a case such as this, in which silent acute cholecystitis was observed, the combined approach was shown to be safe.

Endovascular treatment of aneurysms is already wellestablished. ${ }^{9}$ It is indicated for patients presenting high surgical risk who are in a good anatomical condition. ${ }^{10} \mathrm{In}$ this case, in addition to presenting these two premises, the patient was waiting for a donor kidney for transplantation. We sought to avoid open procedures to avoid surgical manipulation of the retroperitoneum and thereby to facilitate the kidney transplantation in the iliac fossa.

Another advantage of this technique is the shorter duration of ileal paralysis, thus making it possible to resume feeding at an early stage. ${ }^{11}$ Our patient began receiving food after 36 hours and was able to walk after only six hours. We chose to do the endovascular treatment first because pneumoperitoneum is not a problem when using a noncompressible metallic endoprosthesis.

\section{REFERENCES}

1. Ouriel K, Riccota JJ, Adams JT, Deweese JA. Management of cholelithiasis in patients with abdominal aortic aneurysm. Ann Surg 1983;198:717-9.

2. VanekVW.Combining abdominal aortic aneurysmectomy with gastrointestinal or biliary surgery. Am Surgeon 1998;54:290-6.

3. Cadot H, Addis MD, Faries PL, Carroccio A, Burks JA Jr, Gravereaux $\mathrm{EC}$, et al. Abdominal aortic aneurysmorrhaphy and cholelithiasis in the era of endovascular surgery. Am Surg. 2002;68:839-43.

4. Wolosker N, Nishinari K, Ferrari FB, Nakano L, Halpern H, PuechLeão P. Infrarenal aortic aneurysm repair by retroperitoneal approach combined with laparoscopic cholecystectomy: two case reports. J Laparoendosc Adv Surg Tech A. 2001;11:115-7.

5. String ST. Cholelithiasis and aortic reconstruction. J Vasc Surg. 1984;1:664-9.
6. Swanson RJ, Littooy FN, Hunt TK, Stoney RJ. Laparotomy as a precipitating factor in the rupture of intra-abdominal aneurysms. Arch Surg. 1980;115:299-304.

7. Orra HA, Puech-Leão P, Silva ES, Silva DG. Aneurysm pulsatility after endovascular exclusion--an experimental study using human aortic aneurysms. Clinics. 2008;63:67-70.

8. das Chagas de Azevedo F, Zerati AE, Blasbalg R, Wolosker N, PuechLeão P. Comparison of ultrasonography, computed tomography, and magnetic ressonance imaging with intraoperative measurements in the evaluation of abdominal aortic aneurysms. Clinics. 2005;60:21-8.

9. Drury D, Michaels JA, Jones L, Ayiku L. Systematic review of recent evidence for the safety and efficacy of elective endovascular repair in the management of infrarenal abdominal aortic aneurysm. Br J Surg. 2005;92:937-46. 